\title{
On the chromatic number of simple triangle-free triple systems
}

\author{
Alan Frieze* $\quad$ Dhruv Mubayi ${ }^{\dagger}$ \\ Submitted: May 17, 2008; Accepted: Sep 12, 2008; Published: Sep 22, 2008 \\ Mathematics Subject Classification: 05D05, 05D40
}

\begin{abstract}
A hypergraph is simple if every two edges share at most one vertex. It is trianglefree if in addition every three pairwise intersecting edges have a vertex in common. We prove that there is an absolute constant $c$ such that the chromatic number of a simple triangle-free triple system with maximum degree $\Delta$ is at most $c \sqrt{\Delta / \log \Delta}$. This extends a result of Johansson about graphs, and is sharp apart from the constant $c$.
\end{abstract}

\section{Introduction}

Many of the recent important developments in extremal combinatorics have been concerned with generalizing well-known basic results in graph theory to hypergraphs. The most famous of these is the generalization of Szemerédi's regularity lemma to hypergraphs and the resulting proofs of removal lemmas and the multidimensional Szemerédi theorem about arithmetic progressions [4,11, 14]. Other examples are the extension of Dirac's theorem on hamilton cycles [13] and the Chvatal-Rödl-Szemerédi-Trotter theorem on Ramsey numbers of bounded degree graphs [9]. In this paper we continue this theme, by generalizing a result about the chromatic number of graphs.

* Department of Mathematical Sciences, Carnegie Mellon University, Pittsburgh PA 15213. Supported in part by NSF Grant CCF-0502793

${ }^{\dagger}$ Department of Mathematics, Statistics, and Computer Science, University of Illinois, Chicago, IL 60607. Supported in part by NSF Grant DMS-0653946

THE ELECTRONiC JOURNAL OF COMBINATORICs 15 (2008), \#R121 
The basic bound on the chromatic number of a graph of maximum degree $\Delta$ is $\Delta+1$ obtained by coloring the vertices greedily; Brooks theorem states that equality holds only for cliques and odd cycles. Taking this further, one may consider imposing additional local constraints on the graph and asking whether the aforementioned bounded decreases. Kahn and Kim [6] conjectured that if the graph is triangle-free, then the upper bound can be improved to $O(\Delta / \log \Delta)$. Kim [7] proved this with the additional hypothesis that $G$ contains no 4-cycle. Soon after, Johansson proved the conjecture.

Theorem 1 (Johansson [5]) There is an absolute constant $c$ such that every trianglefree graph with maximum degree $\Delta$ has chromatic number at most $c \Delta / \log \Delta$.

It is well known that Theorem 1 is sharp apart from the constant $c$, and Johansson's result was considered a major breakthrough. We prove a similar result for hypergraphs. For $k \geq 2$, a $k$-uniform hypergraph ( $k$-graph for short) is a hypergraph whose edges all have size $k$. A proper coloring of a $k$-graph is a coloring of its vertices such that no edge is monochromatic, and the chromatic number is the minimum number of colors in a proper coloring. An easy consequence of the Local Lemma is that every 3-graph with maximum degree $\Delta$ has chromatic number at most $3 \sqrt{\Delta}$. Our result improves this if we impose local constraints on the 3-graph. Say that a $k$-graph is simple if every two edges share at most one vertex. A triangle in a simple $k$-graph is a collection of three pairwise intersecting edges containing no common point. We extend Johansson's theorem to hypergraphs as follows.

Theorem 2 There are absolute positive constants $c, c^{\prime}$ such that the following holds: Every simple triangle-free 3-graph with maximum degree $\Delta$ has chromatic number at most $c \sqrt{\Delta / \log \Delta}$. Moreover, there exist simple triangle-free 3-graphs with maximum degree $\Delta$ and chromatic number at least $c^{\prime} \sqrt{\Delta / \log \Delta}$.

Theorem 2 can also be considered as a generalization of a classical result of Komlos-PintzSzemerédi [8] who proved, under the additional hypotheses that there are no 4-cycles, that triple systems with $n$ vertices and maximum degree $\Delta$ have an independent set of size at least $c\left(n / \Delta^{1 / 2}\right)(\log \Delta)^{1 / 2}$ where $c$ is a constant.

Simple hypergraphs share many of the complexities of (more general) hypergraphs but also have many similarities with graphs. We believe that Theorem 2 can be proved for general 3-graphs, but the proof would probably require several new ideas. Our argument uses simplicity in several places (see Section 11). In fact, we conjecture that a similar 
result holds for $k$-graphs as long as any fixed subhypergraph is forbidden. The analogous conjecture for graphs was posed by Alon-Krivelevich-Sudakov [2].

Conjecture 3 Let $F$ be a k-graph. There is a constant $c_{F}$ depending only on $F$ such that every $F$-free $k$-graph with maximum degree $\Delta$ has chromatic number at most $c_{F}(\Delta / \log \Delta)^{1 /(k-1)}$.

Note that this Conjecture implies that the upper bound in Theorem 2 holds even if we exclude the triangle-free hypothesis ${ }^{1}$. Indeed, the condition of simplicity is the same as saying that the 3 -graph is $F$-free, where $F$ is the 3 -graph of two edges sharing two vertices. The proof of the lower bound in Theorem 2 is fairly standard. The idea is to take a random $k$-graph with appropriate edge probability, and then cleverly delete all copies of triangles from it. This approach was used by Krivelevich [10] to prove lower bounds for off diagonal Ramsey numbers. More recently, it was extended to families of hypergraphs in [3] and we will use this result.

The proof of the upper bound in Theorem 2 is our main contribution. Here we will heavily expand on ideas used by Johansson in his proof of Theorem 1. The approach, which has been termed the semi-random, or nibble method, was first used by Rödl (although his proof was inspired by earlier work in [1]) to settle the Erdős-Hanani conjecture about the existence of asymptotically optimal designs. Subsequently, inspired by work of Kahn [6], Kim [7] proved Theorem 1 for graphs with girth five. Finally Johansson using a host of additional ideas, proved his result. The approach used by Johansson for the graph case is to iteratively color a small portion of the (currently uncolored) vertices of the graph, record the fact that a color already used at $v$ cannot be used in future on the uncolored neighbors of $v$, and continue this process until the graph induced by the uncolored vertices has small maximum degree. Once this has been achieved, the remaining uncolored vertices are colored using a new set of colors by the greedy algorithm. Since the initial maximum degree is $\Delta$, we require that the final degree is of order $\Delta / \log \Delta$ in order for the greedy algorithm to be efficient. At each step, the degree at each vertex will fall roughly by a multiplicative factor of $(1-1 / \log \Delta)$, and so the number of steps in the semi random phase of the algorithm is roughly $\log \Delta \log \log \Delta$.

In principle our method is the same, but there are several difficulties we encounter. The first, and most important, is that our coloring algorithm must necessarily be more complicated. A proper coloring of a 3-graph allows two vertices of an edge to have the same

\footnotetext{
${ }^{1}$ The authors have recently proved this particular special case of Conjecture 3 for arbitrary $k \geq 3$.
} 
color, indeed, to obtain optimal results one must permit this. To facilitate this, we introduce a graph at each stage of our algorithm whose edges comprise pairs of uncolored vertices that form an edge of the 3-graph with a colored vertex. Keeping track of this graph requires controlling more parameters during the iteration and dealing with some more lack of independence and this makes the proof more complicated. Finally, we remark that our theorem also proves the same upper bound for list chromatic number, although we phrase it only for chromatic number.

In the next section we present the lower bound in Theorem 2 and the rest of the paper is devoted to the proof of the upper bound. The last section describes the minor modifications to the main argument that would yield the corresponding result for list colorings.

\section{Random construction}

In this section we prove the lower bound in Theorem 2. We will actually observe that a slightly more general result follows from a theorem in [3]. Let us begin with a definition. Call a hypergraph nontrivial if it has at least two edges.

Definition 4 Let $F$ be a nontrivial k-graph. Then

$$
\rho(F)=\max _{F^{\prime} \subset F} \frac{e^{\prime}-1}{v^{\prime}-k}
$$

where $F^{\prime}$ is nontrivial with $v^{\prime}$ vertices and $e^{\prime}$ edges. For a finite family $\mathcal{F}$ of nontrivial $k$-graphs, $\rho(\mathcal{F})=\min _{F \in \mathcal{F}} \rho(F)$.

Theorem 5 Let $\mathcal{F}$ be a finite family of nontrivial $k$-graphs with $\rho(\mathcal{F})>1 /(k-1)$. There is an absolute constant $c=c_{\mathcal{F}}$ such that the following holds: for all $\Delta>0$, there is an $\mathcal{F}$-free $k$-graph with maximum degree $\Delta$ and chromatic number at least $c(\Delta / \log \Delta)^{1 /(k-1)}$.

Proof. Fix $k \geq 2$ and let $\rho=\rho(\mathcal{F})$. Consider the random $k$-graph $G_{p}$ with vertex set $[n]$ and each edge appearing independently with probability $p=n^{-1 / \rho}$. Then an easy calculation using the Chernoff bounds shows that with probability tending to 1 , the maximum degree $\Delta$ of $G$ satisfies $\Delta<n^{k-1-1 / \rho}$. Let us now delete the edges of a maximal collection of edge disjoint copies of members of $\mathcal{F}$ from $G_{p}$. The resulting $k$-graph $G_{p}^{\prime}$ is clearly $\mathcal{F}$-free. Moreover, it is shown in [3] that with probability tending to 1 , the maximum size $t$ of an independent set of vertices in $G_{p}^{\prime}$ satisfies

$$
t<c_{1}\left(n^{1 / \rho} \log n\right)^{1 /(k-1)}
$$


where $c_{1}$ depends only on $\mathcal{F}$. Consequently, the chromatic number of $G_{p}^{\prime}$ is at least

$$
c_{2}\left(\frac{n^{k-1-1 / \rho}}{\log n}\right)^{1 /(k-1)}>c_{3}\left(\frac{\Delta}{\log \Delta}\right)^{1 /(k-1)},
$$

where $c_{2}$ and $c_{3}$ depend only on $\mathcal{F}$. This completes the proof.

The lower bound in Theorem 2 is an easy consequence of Theorem 5 . Indeed, let $k=3$ and $\mathcal{F}=\left\{F_{1}, F_{2}\right\}$, where $F_{1}$ is the 3 -graph of two edges sharing two vertices, and $F_{2}$ is a simple triangle i.e. $F_{2}=\{a b c, c d e$, efa $\}$. Then $\rho\left(F_{1}\right)=1$ and $\rho\left(F_{2}\right)=2 / 3$ so they are both greater than $1 / 2$ and Theorem 5 applies.

\section{Local Lemma}

The driving force of our upper bound argument, both in the semi-random phase and the final phase, is the Local Lemma. We use it in the form below.

Theorem 6 (Local Lemma) Let $\mathcal{A}_{1}, \ldots, \mathcal{A}_{n}$ be events in an arbitrary probability space. Suppose that each event $\mathcal{A}_{i}$ is mutually independent of a set of all the other events $\mathcal{A}_{j}$ but at most $d$, and that $P\left(\mathcal{A}_{i}\right)<p$ for all $1 \leq i \leq n$. If ep $(d+1)<1$, then with positive probability, none of the events $\mathcal{A}_{i}$ holds.

Note that the Local Lemma immediately implies that every 3-graph with maximum degree $\Delta$ can be properly colored with at most $\lceil 3 \sqrt{\Delta}\rceil$ colors. Indeed, if we color each vertex randomly and independently with one of these colors, the probability of the event $\mathcal{A}_{e}$, that an edge $e$ is monochromatic, is at most $1 / 9 \Delta$. Moreover $\mathcal{A}_{e}$ is independent of all other events $\mathcal{A}_{f}$ unless $|f \cap e|>0$, and the number of $f$ satisfying this is less than $3 \Delta$. We conclude that there is a proper coloring.

\section{Coloring Procedure}

In the rest of the paper, we will prove the upper bound in Theorem 2. Suppose that $H$ is a simple triangle-free 3 -graph with maximum degree $\Delta$. We will assume that $\Delta$ is sufficiently large that all implied inequalities below hold true. Also, all asymptotic notation should be taken as $\Delta \rightarrow \infty$. Let $V$ be the vertex set of $H$. As usual, we write $\chi(H)$ for the chromatic number of $H$. Let $\varepsilon>0$ be a sufficiently small fixed number. Throughout the paper, we will omit the use of floor and ceiling symbols. 
Let

$$
q=\frac{\Delta^{1 / 2}}{\omega^{1 / 2}}
$$

where

$$
\omega=\frac{\varepsilon \log \Delta}{10^{4}}
$$

We color $V$ with $2 q$ colors and therefore show that

$$
\chi(H) \leq \frac{200}{\varepsilon^{1 / 2}} \frac{\Delta^{1 / 2}}{(\log \Delta)^{1 / 2}} .
$$

We use the first $q$ colors to color $H$ in rounds and then use the second $q$ colors to color any vertices not colored by this process.

Our algorithm for coloring in rounds is semi-random. At the beginning of a round certain parameters will satisfy certain properties, (6) - (11) below. We describe a set of random choices for the parameters in the next round and we use the local lemma to prove that there is a set of choices that preserves the required properties.

- $C=[q]$ denotes the set of available colors for the semi-random phase.

- $U^{(t)}$ : The set of vertices which are currently uncolored. $\left(U^{(0)}=V\right)$.

- $H^{(t)}$ : The sub-hypergraph of $H$ induced by $U^{(t)}$.

- $W^{(t)}=V \backslash U^{(t)}$ : The set of vertices that have been colored. We use the notation $\kappa$ to denote the color of an item e.g. $\kappa(w), w \in W^{(t)}$ denotes the color permanently assigned to $w$.

- $G^{(t)}$ : An edge-colored graph with vertex set $U^{(t)}$. There is an edge $u v \in G^{(t)}$ iff there is a vertex $w \in W^{(t)}$ and an edge $u v w \in H$. Because $H$ is simple, $w$ is unique, if it exists. The edge $u v$ is given the color $\kappa(u v)=\kappa(w)$. (This graph is used to keep track of some coloring restrictions).

- $p_{u}^{(t)} \in[0,1]^{C}$ for $u \in U^{(t)}$ : This is a vector of coloring probabilities. The $c$ th coordinate is denoted by $p_{u}^{(t)}(c)$ and $p_{u}^{(0)}=\left(q^{-1}, q^{-1}, \ldots, q^{-1}\right)$.

We can now describe the "algorithm" for computing $U^{(t+1)}, p_{u}^{(t+1)}, u \in U^{(t+1)}$ etc., given $U^{(t)}, p_{u}^{(t)}, u \in U^{(t)}$ etc.: Let

$$
\theta=\frac{\varepsilon}{\omega}=\frac{10^{4}}{\log \Delta}
$$

where we recall that $\varepsilon$ is a sufficiently small positive constant. 
For each $u \in U^{(t)}$ and $c \in C$ we tentatively activate $c$ at $u$ with probability $\theta p_{u}^{(t)}(c)$. A color $c$ is lost at $u \in U^{(t)}, p_{u}^{(t+1)}(c)=0$ and $p_{u}^{\left(t^{\prime}\right)}(c)=0$ for $t^{\prime}>t$ if there is an edge $u v w \in H^{(t)}$ such that $c$ is tentatively activated at $v$ and $w$. In addition, a color $c$ is lost at $u \in U^{(t)}$ if there is an edge $u v \in G^{(t)}$ such that $c$ is tentatively activated at $v$ and $\kappa(u v)=c$.

The vertex $u \in U^{(t)}$ is given a permanent color if there is a color tentatively activated at $u$ which is not lost due to the above reasons. If there is a choice, it is made arbitrarily. Then $u$ is placed into $W^{(t+1)}$.

We fix

$$
\hat{p}=\frac{1}{\Delta^{11 / 24}}
$$

(We can replace $11 / 24$ by any $\alpha \in(5 / 12,1 / 2)$ ).

We keep

$$
p_{u}^{(t)}(c) \leq \hat{p}
$$

for all $t, u, c$.

We let

$$
B^{(t)}(u)=\left\{c: p_{u}^{(t)}(c)=\hat{p}\right\} \quad \text { for all } u \in V .
$$

A color in $B^{(t)}(u)$ cannot be used at $u$. The role of $B^{(t)}(u)$ is clarified later.

Here are some more details:

\section{Coloring Procedure: Round $t$}

Make tentative random color choices

Independently, for all $u \in U^{(t)}, c \in C$, let

$$
\begin{gathered}
\gamma_{u}^{(t)}(c)= \begin{cases}1 & \text { Probability }=\theta p_{u}^{(t)}(c) \\
0 & \text { Probability }=1-\theta p_{u}^{(t)}(c)\end{cases} \\
\Theta^{(t)}(u)=\left\{c: \gamma_{u}^{(t)}(c)=1\right\}=\text { the set of colors tentatively activated at } u .
\end{gathered}
$$

\section{Deal with color clashes}

$$
\begin{aligned}
& L^{(t)}(u)=\left\{c: \exists u v w \in H^{(t)} \text { such that } c \in \Theta^{(t)}(v) \cap \Theta^{(t)}(w)\right\} \cup \\
& \left\{c: \exists u v \in G^{(t)} \text { such that } \kappa(u v)=c \in \Theta^{(t)}(v)\right\}
\end{aligned}
$$

is the set of colors lost at $u$ in this round.

$$
A^{(t)}(u)=A^{(t-1)}(u) \cup L^{(t)}(u) .
$$




\section{Assign some permanent colors}

Let

$\Psi^{(t)}(u)=\Theta^{(t)}(u) \backslash\left(A^{(t)}(u) \cup B^{(t)}(u)\right)=$ set of activated colors that can be used at $u$.

If $\Psi^{(t)}(u) \neq \emptyset$ then choose $c \in \Psi^{(t)}(u)$ arbitrarily. Let $\kappa(u)=c$.

We now describe how to update the various parameters:

(a)

$$
U^{(t+1)}=U^{(t)} \backslash\left\{u: \Psi^{(t)}(u) \neq \emptyset\right\}
$$

(b) $G^{(t+1)}$ is the graph with vertex set $U^{(t+1)}$ and edges $\left\{u v: \exists u v w \in H, w \notin U^{(t+1)}\right\}$. Edge $u v$ has color $\kappa(u v)=\kappa(w)$. (H simple implies that there is at most one $w$ for any $u v)$.

(c) $p_{u}^{(t)}(c)$ is replaced by a random value $p_{u}^{\prime}(c)$ which is either 0 or at least $p_{u}^{(t)}(c)$. Furthermore, if $u \in U^{(t)} \backslash U^{(t+1)}$ then by convention $p_{u}^{\left(t^{\prime}\right)}=p_{u}^{(t+1)}$ for all $t^{\prime}>t$. The key property is

$$
\mathrm{E}\left(p_{u}^{\prime}(c)\right)=p_{u}^{(t)}(c)
$$

The update rule is as follows: If $c \in A^{(t-1)}(u)$ then $p_{u}^{(t)}(c)$ remains unchanged at zero. Otherwise,

$$
p_{u}^{\prime}(c)=\left\{\begin{array}{lll}
\left\{\begin{array}{lll}
0 & c \in L^{(t)}(u) & \frac{p_{u}^{(t)}(c)}{q_{u}^{(t)}(c)}<\hat{p}
\end{array}\right. & \text { Case A } \\
\frac{p_{u}^{(t)}(c)}{q_{u}^{(t)}(c)} & c \notin L^{(t)}(u) & \\
\eta_{u}^{(t)}(c) \hat{p} & \frac{p_{u}^{(t)}(c)}{q_{u}^{(t)}(c)} \geq \hat{p} & \text { Case B },
\end{array}\right.
$$

where

$$
q_{u}^{(t)}(c)=\prod_{u v w \in H^{(t)}}\left(1-\theta^{2} p_{v}^{(t)}(c) p_{w}^{(t)}(c)\right) \prod_{\substack{u v \in G^{(t)} \\ \kappa(u v)=c}}\left(1-\theta p_{v}^{(t)}(c)\right)
$$

is the probability that $c \notin A^{(t)}(u)$ assuming that $c \notin A^{(t-1)}(u)$.

- $\eta_{u}^{(t)}(c) \in\{0,1\}$ and $\mathrm{P}\left(\eta_{u}^{(t)}(c)=1\right)=p_{u}^{(t)}(c) / \hat{p}$, independently of other variables. 
Remark $\mathbf{7}$ It is as well to remark here that the probability space on which our events for iteration $t$ are defined is a product space where each component corresponds to $\gamma_{u}^{(t)}(c)$ or $\eta_{u}^{(t)}(c)$ for $u \in V, c \in C$. Hopefully, this will provide the reader with a clear understanding of the probabilities involved below.

There will be

$$
t_{0}=\varepsilon^{-1} \log \Delta \log \log \Delta \text { rounds. }
$$

Before getting into the main body of the proof, we check (2). If $p_{u}^{(t)}(c) / q_{u}^{(t)}(c)<\hat{p}$ then

$$
\mathrm{E}\left(p_{u}^{\prime}(c)\right)=q_{u}^{(t)}(c) \frac{p_{u}^{(t)}(c)}{q_{u}^{(t)}(c)}=p_{u}^{(t)}(c)
$$

If $p_{u}^{(t)}(c) / q_{u}^{(t)}(c) \geq \hat{p}$ then

$$
\mathrm{E}\left(p_{u}^{\prime}(c)\right)=\hat{p} \frac{p_{u}^{(t)}(c)}{\hat{p}}=p_{u}^{(t)}(c) .
$$

Note that once a color enters $B^{(t)}(u)$, it will be in $B^{\left(t^{\prime}\right)}(u)$ for all $t^{\prime} \geq t$. This is because we update $p_{u}(c)$ according to Case $\mathrm{B}$ and $\mathrm{P}\left(\eta_{u}^{(t)}(c)=1\right)=1$. We arrange things this way, because we want to maintain $(2)$. Then because $p_{u}^{(t)}(c)$ cannot exceed $\hat{p}$, it must actually remain at $\hat{p}$. This could cause some problems for us if a neighbor of $u$ had been colored with $c$. This is why $B^{(t)}(u)$ is excluded in the definition of $\Psi^{(t)}(u)$ i.e. we cannot color $u$ with $c \in B^{(t)}(u)$.

\section{Correctness of the coloring}

Observe that if color $c$ enters $A^{(t)}(x)$ at some time $t$ then $\kappa(x) \neq c$ since $A^{(i)}(x) \subseteq A^{(i+1)}(x)$ for all $i$. Suppose that some edge uvw is improperly colored by the above algorithm. Suppose that $u, v, w$ get colored at times $t_{u} \leq t_{v} \leq t_{w}$ and that $\kappa(u)=\kappa(v)=\kappa(w)=c$. If $t_{u}=t_{v}=t$ then $c \in L^{(t)}(w)$ and so $\kappa(w) \neq c$. If $t_{u}<t_{v}=t$ then $v w$ is an edge of $G^{(t)}$ and $\kappa(v w)=c$ and so $c \in L^{(t)}(w)$ and again $\kappa(w) \neq c$.

\section{Parameters for the problem}

We will now drop the superscript $(t)$, unless we feel it necessary. It will be implicit i.e. $p_{u}(c)=p_{u}^{(t)}(c)$ etcetera. Furthermore, we use $\mathrm{a}^{\prime}$ to replace the superscript $(t+1)$ i.e. 
$p_{u}^{\prime}(c)=p_{u}^{(t+1)}(c)$ etcetera. The following are the main parameters that we need in the course of the proof:

$$
\begin{aligned}
e_{u v w} & =\sum_{c \in C} p_{u}(c) p_{v}(c) p_{w}(c) \quad \text { for edge uvw of } H^{(t)} . \\
f_{u} & =\sum_{c \in C} \sum_{u v \in G} 1_{\kappa(u v=c)} p_{u}(c) p_{v}(c) \\
h_{u} & =-\sum_{c \in C} p_{u}(c) \log p_{u}(c) . \\
d_{G}(u, c) & =\mid\{v: u v \in G \text { and } \kappa(u v)=c\} \mid \\
d_{G}(u) & =\sum_{c \in C} d_{G}(u, c) \quad=\text { degree of u in } G \\
d_{H^{(t)}}(u) & =\mid\left\{v w: \text { uvw } \in H^{(t)}\right\} \mid=\text { degree of u in } H^{(t)} \\
d(u) & =d_{G}(u)+d_{H^{(t)}}(u)
\end{aligned}
$$

It will also be convenient to define the following auxiliary parameters:

$$
\begin{aligned}
e_{u} & =\sum_{u v w \in H^{(t)}} e_{u v w} \\
e_{v w}(c) & =p_{v}(c) p_{w}(c) \\
e_{u}(c) & =\sum_{u v w \in H^{(t)}} e_{v w}(c) \\
f_{u}(c) & =\sum_{\{u v \in G: \kappa(u v)=c\}} p_{v}(c)
\end{aligned}
$$

This gives

$$
\begin{aligned}
e_{u} & =\sum_{c \in C} p_{u}(c) e_{u}(c) \\
f_{u} & =\sum_{c \in C} p_{u}(c) f_{u}(c) .
\end{aligned}
$$

\section{Invariants}

Following Johansson [5], we define a set of properties such that if they are satisfied at time $t$ then it is possible to extend our partial coloring and maintain these properties at time $t+1$. These properties are now listed. They are only claimed for $u \in U$. 


$$
\begin{aligned}
\left|1-\sum_{c} p_{u}(c)\right| & \leq t \Delta^{-1 / 8} . \\
e_{u v w} & \leq e_{u v w}^{(0)}+\frac{t}{\Delta^{10 / 9}} \quad \forall u v w \in H^{(t)} \\
& \leq \frac{\omega}{\Delta}+\frac{t}{\Delta^{10 / 9}} . \\
f_{u} & \leq 3(1-\theta / 4)^{t} \omega . \\
h_{u} & \geq h_{u}^{(0)}-5 \varepsilon \sum_{i=0}^{t}(1-\theta / 4)^{i} . \\
d(u) & \leq\left(1-\frac{\theta}{3}\right)^{t} \Delta . \\
d_{G}(u, c) & \leq 2 t \theta \Delta \hat{p} .
\end{aligned}
$$

Equation (10) shows that after $t_{0}$ rounds we find that the maximum degree in the hypergraph induced by the uncolored vertices satisfies

$$
\begin{aligned}
\Delta\left(H^{\left(t_{0}\right)}\right) & \leq\left(1-\frac{\theta}{3}\right)^{t_{0}} \Delta \\
& \leq e^{-\theta t_{0} / 3} \Delta \\
& <e^{-4000 \log \log \Delta} \Delta \\
& =\frac{\Delta}{(\log \Delta)^{4000}} .
\end{aligned}
$$

and then the local lemma (see the argument after Theorem 6) will show that the remaining vertices can be colored with a set of $3\left(\Delta /(\log \Delta)^{4000}\right)^{1 / 2}+1<q$ new colors. 


\section{Dynamics}

To prove $(6)-(11)$ we show that we can find updated parameters such that

$$
\begin{aligned}
\left|\sum_{c} p_{u}^{\prime}(c)-\sum_{c} p_{u}(c)\right| & \leq \Delta^{-1 / 8} \\
e_{u v w}^{\prime} & \leq e_{u v w}+\Delta^{-10 / 9} . \\
f_{u}^{\prime}-f_{u} & \leq \theta\left(2 e_{u}-(1-7 \varepsilon) f_{u}\right)+\Delta^{-1 / 21} . \\
h_{u}-h_{u}^{\prime} & \leq 5 \varepsilon(1-\theta / 4)^{t} \\
d^{\prime}(u) & \leq(1-3 \theta / 7) d(u)+\Delta^{2 / 3} . \\
d_{G^{\prime}}(u, c) & \leq d_{G}(u, c)+2 \theta \Delta \hat{p}
\end{aligned}
$$

\section{9 (13)-(18) imply (6)-(11)}

First let us show that (13)-(18) are enough to inductively prove that (6)-(10) hold throughout.

Property (6): Trivial.

Property (7): Trivial.

Property (8): Fix $u$ and note that (7) and (10) imply

$$
e_{u} \leq\left(\frac{\omega}{\Delta}+t \Delta^{-10 / 9}\right) d(u) \leq \omega(1-\theta / 3)^{t}+\Delta^{-1 / 10} .
$$

Therefore,

$$
f_{u}^{\prime}-f_{u} \leq \theta\left(2 \omega(1-\theta / 3)^{t}+\Delta^{-1 / 22}-(1-7 \varepsilon) f_{u}\right)
$$

from (15) and (19). So, using $f_{u} \leq 3(1-\theta / 4)^{t} \omega$,

$$
\begin{aligned}
f_{u}^{\prime} & \leq 3(1-\theta(1-7 \varepsilon))(1-\theta / 4)^{t} \omega+2 \theta \omega(1-\theta / 3)^{t}+\theta \Delta^{-1 / 22} \\
& =3(1-\theta / 4)^{t+1} \omega+\omega(1-\theta / 4)^{t}\left(-3 \theta(1-7 \varepsilon)+2 \theta\left(\frac{1-\theta / 3}{1-\theta / 4}\right)^{t}\right)+\theta \Delta^{-1 / 22} \\
& \leq 3(1-\theta / 4)^{t+1} \omega+\omega(1-\theta / 4)^{t}(-3 \theta(1-7 \varepsilon)+2 \theta)+\theta \Delta^{-1 / 22} \\
& \leq 3(1-\theta / 4)^{t+1} \omega-\omega \theta(1-21 \varepsilon)(\log \Delta)^{-O(1)}+\theta \Delta^{-1 / 22} \\
& \leq 3(1-\theta / 4)^{t+1} \omega .
\end{aligned}
$$

Property (9): Trivial. 
Property (10): If $d(u) \leq(1-\theta / 3)^{t} \Delta$ then from (17) we get

$$
\begin{aligned}
d^{\prime}(u) & \leq\left(1-\frac{3 \theta}{7}\right)\left(1-\frac{\theta}{3}\right)^{t} \Delta+\Delta^{2 / 3} \\
& =\left(1-\frac{\theta}{3}\right)^{t+1} \Delta-\frac{2 \theta}{21}\left(1-\frac{\theta}{3}\right)^{t} \Delta+\Delta^{2 / 3} \\
& \leq\left(1-\frac{\theta}{3}\right)^{t+1} \Delta .
\end{aligned}
$$

Property (11): Trivial.

To complete the proof it suffices to show that there are choices for $\gamma_{u}(c), \eta_{u}(c), u \in U, c \in$ $C$ such that (13)-(18) hold.

In order to help understand the following computations, the reader is reminded that quantities $e_{u}, f_{u}, \omega, \theta^{-1}$ can all be upper bounded by $\Delta^{o(1)}$.

\section{Bad colors}

We now put a bound on the weight of the colors in $B(u)$.

Assume that (6)-(10) hold. It follows from (9) that

$$
h_{u}^{(0)}-h_{u}^{(t)} \leq 5 \varepsilon \sum_{i=0}^{\infty}(1-\theta / 4)^{i}=20 \omega=\frac{\varepsilon \log \Delta}{500} .
$$

Since $p_{u}^{(0)}(c)=1 / q$ for all $u, c$ we have

$$
\begin{aligned}
h_{u}^{(0)} & =-\sum_{c} p_{u}^{(0)}(c) \log p_{u}^{(0)}(c) \\
& =-\sum_{c} p_{u}^{(t)}(c) \log p_{u}^{(0)}(c)-(\log 1 / q) \sum_{c}\left(p_{u}^{(0)}(c)-p_{u}^{(t)}(c)\right) \\
& \geq-\sum_{c} p_{u}^{(t)}(c) \log p_{u}^{(0)}(c)-t \Delta^{-1 / 9} \log \Delta .
\end{aligned}
$$

where the last inequality uses (6).

Plugging this lower bound on $h_{u}^{(0)}$ into (20) gives

$$
\sum_{c} p_{u}^{(t)}(c) \log \left(p_{u}^{(t)}(c) / p_{u}^{(0)}(c)\right) \leq \frac{\varepsilon \log \Delta}{500}+\Delta^{-1 / 10}
$$


Now, all terms in $(21)$ are non-negative $\left(p_{u}^{(t)}(c)=0\right.$ or $\left.p_{u}^{(t)}(c) \geq p_{u}^{(0)}(c)\right)$. Thus after dropping the contributions from $c \notin B(u)$ we get

$$
\begin{aligned}
\frac{\varepsilon \log \Delta}{500}+\Delta^{-1 / 10} & \geq \sum_{c \in B(u)} p_{u}^{(t)}(c) \log \left(p_{u}^{(t)}(c) / p_{u}^{(0)}(c)\right) \\
& =\sum_{c \in B(u)} p_{u}^{(t)}(c) \log (\hat{p} q)=\sum_{c \in B(u)} p_{u}^{(t)}(c) \log \left(\Delta^{1 / 24-o(1)}\right) \\
& \geq \frac{1}{25} p_{u}(B(u)) \log \Delta .
\end{aligned}
$$

So,

$$
p_{u}(B(u)) \leq \frac{\varepsilon}{10}
$$

\section{Verification of Dynamics}

Let $\mathcal{E}_{13}(u)-\mathcal{E}_{18}(u)$ be the events claimed in equations $(13)-(18)$. Let $\mathcal{E}(u)=\mathcal{E}_{13}(u) \cap$ $\cdots \cap \mathcal{E}_{18}(u)$. We have to show that $\bigcap_{u \in U} \mathcal{E}(u)$ has positive probability. We use the local lemma. The dependency graph of the $\mathcal{E}(u), u \in U$ has maximum degree $\Delta^{O(1)}$ and so it is enough to show that each event $\mathcal{E}_{13}(u), \ldots, \mathcal{E}_{18}(u), u \in U$ has failure probability $e^{-\Delta^{\Omega(1)}}$. While parameters $e_{u}, f_{u}$ etc. are only needed for $u \in U$ we do not for example consider $e_{u}^{\prime}$ conditional on $u \in U^{\prime}$. We do not impose this conditioning and so we do not have to deal with it. Thus the local lemma will guarantee a value for $e_{u}, u \in U \backslash U^{\prime}$ and we are free to disregard it for the next round.

In the following we will use various forms of Hoeffding's inequality for sums of bounded random variables: We will use it in two forms: Suppose first that $X_{1}, X_{2}, \ldots, X_{m}$ are independent random variables and $\left|X_{i}\right| \leq a_{i}$ for $1 \leq i \leq m$. Let $X=X_{1}+X_{2}+\cdots+X_{m}$. Then, for any $t>0$,

$$
\max \{\mathrm{P}(X-\mathrm{E}(X) \geq t), \mathrm{P}(X-\mathrm{E}(X) \leq-t)\} \leq \exp \left\{-\frac{2 t^{2}}{\sum_{i=1}^{m} a_{i}^{2}}\right\} .
$$

We will also need the following version in the special case that $X_{1}, X_{2}, \ldots, X_{m}$ are independent 0,1 random variables. For $\alpha>1$ we have

$$
\mathrm{P}(X \geq \alpha \mathrm{E}(X)) \leq(e / \alpha)^{\alpha \mathrm{E}(X)} .
$$

\subsection{Dependencies}

In our random experiment, we start with the $p_{u}(c)$ 's and then we instantiate the independent random variables $\gamma_{u}(c), \eta_{u}(c), u \in U, c \in C$ and then we compute the $p_{u}^{\prime}(c)$ from 
these values. Observe first that $p_{u}^{\prime}(c)$ depends only on $\gamma_{v}(c), \eta_{v}(c)$ for $v=u$ or $v$ a neighbor of $u$ in $H$. So $p_{u}^{\prime}(c)$ and $p_{v}^{\prime}\left(c^{*}\right)$ are independent if $c \neq c^{*}$, even if $u=v$. We call this color independence.

Let

$$
N(u)=\{v \in U: \exists u v w \in H\} .
$$

(We do mean $H$ and not $H^{(t)}$ here).

Observe that by repeatedly using $(1-a)(1-b) \geq 1-a-b$ for $a, b \geq 0$ we see that

$$
q_{u}(c) \geq 1-\theta^{2} e_{u}(c)-\theta f_{u}(c) .
$$

This inequality will be used below. Recall that $1-q_{u}(c)$ is the probability that $c$ will be placed in $L(u)$ in the current round.

For each $v \in N(u)$ we let

$$
C_{u}(v)=\left\{c \in C: \gamma_{u}(c)=1\right\} \cup L(v) \cup B(v) .
$$

Note that while the first two sets in this union depend on the random choices made in this round, the set $B(v)$ is already defined at the beginning of the round.

We will later use the fact that if $c^{*} \notin C_{u}(v)$ and $\gamma_{v}\left(c^{*}\right)=1$ then this is enough to place $c^{*}$ into $\Psi(v)$ and allow $v$ to be colored. Indeed, $\gamma_{v}\left(c^{*}\right)=1$ implies that $p_{v}(c) \neq 0$ from which it follows that $c^{*} \notin A(v)$.

Let $Y_{v}=\sum_{c} p_{v}(c) 1_{c \in C_{u}(v)}=p_{v}\left(C_{u}(v)\right)$. $C_{u}(v)$ is a random set and $Y_{v}$ is the sum of $q$ independent random variables each one bounded by $\hat{p}$. Then by (4), (5) and (25),

$$
\begin{aligned}
\mathrm{E}\left(Y_{v}\right) & \leq \sum_{c \in C} p_{v}(c) \mathrm{P}\left(\gamma_{u}(c)=1\right)+\sum_{c \in C} p_{v}(c)\left(1-q_{v}(c)\right)+p_{v}(B(v)) \\
& \leq \theta \sum_{c \in C} p_{u}(c) p_{v}(c)+\theta^{2} e_{v}+\theta f_{v}+p_{v}(B(v)) .
\end{aligned}
$$

Now let us bound each term separately:

$$
\theta \sum_{c \in C} p_{u}(c) p_{v}(c) \leq \theta q \hat{p}^{2}<\theta \Delta^{1 / 2} \Delta^{-11 / 12}<\frac{10^{4} \Delta^{-5 / 12}}{\log \Delta}<\frac{\varepsilon}{3} .
$$

Using (7) we obtain

$$
\theta^{2} e_{v}<\omega \theta^{2}+t \theta^{2} \Delta^{-1 / 9} \leq \varepsilon \theta+t \theta^{2} \Delta^{-1 / 9}<\frac{\varepsilon}{6}+\frac{\varepsilon}{6}=\frac{\varepsilon}{3} .
$$

Using (8) we obtain

$$
\theta f_{v} \leq 3 \theta(1-\theta / 4)^{t} \omega<3 \theta \omega=3 \varepsilon
$$


Together with $\mathrm{P}(B(v)) \leq \varepsilon / 10$ we get

$$
\mathrm{E}\left(Y_{v}\right) \leq 4 \varepsilon
$$

Hoeffding's inequality then gives

$$
\mathrm{P}\left(Y_{v} \geq \mathrm{E}\left(Y_{v}\right)+\rho\right) \leq \exp \left\{-\frac{2 \rho^{2}}{q \hat{p}^{2}}\right\}=e^{-2 \rho^{2} \Delta^{11 / 12-1 / 2-o(1)}} .
$$

Taking $\rho=\Delta^{-1 / 6}$ say, it follows that

$$
\mathrm{P}\left(p_{v}\left(C_{u}(v)\right) \geq 5 \varepsilon\right)=\mathrm{P}\left(Y_{v} \geq 5 \varepsilon\right) \leq e^{-\Delta^{1 / 12-o(1)}} .
$$

Let $\mathcal{E}_{(26)}$ be the event $\left\{p_{v}\left(C_{u}(v)\right) \leq 5 \varepsilon\right\}$.

Now consider some fixed vertex $u \in U$. It will sometimes be convenient to condition on the values $\gamma_{x}(c), \eta_{x}(c)$ for all $c \in C$ and all $x \notin N(u)$ and for $x=u$. This conditioning is needed to obtain independence. We let $\mathcal{C}$ denote these conditional values.

Note that $\mathcal{C}$ determines whether or not $\mathcal{E}_{(26)}$ occurs. (Note that if uvw is an edge of $H$ then $L(v)$ depends on $\gamma_{w}$. We have however made $\left\{c \in C: \gamma_{u}(c)=1\right\}$ part of $C_{u}(v)$ and this removes the dependence of $C_{u}(v)$ on $\left.\gamma_{w}\right)$.

Given the conditioning $\mathcal{C}$, simplicity and triangle freeness imply that the events $\left\{v \notin U^{\prime}\right\}$, $\left\{w \notin U^{\prime}\right\}$ for $v, w \in N(u)$ are independent provided $u v w \notin H$. Indeed, triangle-freeness implies that for $u v w \notin H$, there is no edge containing both $v$ and $w$. Therefore the random choices at $w$ will not affect the coloring of $v$ (and vice versa). Thus random variables $p_{v}^{\prime}(c), p_{w}^{\prime}(c)$ will become (conditionally) independent under these circumstances. We call this conditional neighborhood independence.

\subsubsection{Some expectations}

Let us fix a color $c$ and an edge $u v w \in H$ (here we mean $H$ and not $H^{(t)}$ ) where $u, v \in U$. In this subsection we will estimate the expectations of $p_{u}^{\prime}(c) p_{v}^{\prime}(c) p_{w}^{\prime}(c)$ when $u v w \in H^{(t)}$ and $e_{u v}^{\prime}(c) \times 1_{u, v \in U^{\prime}}$ when $u v \in G$ and $\kappa(u v)=c$.

Estimate for $\mathbf{E}\left(\boldsymbol{p}_{\boldsymbol{u}}^{\prime}(\boldsymbol{c}) \boldsymbol{p}_{\boldsymbol{v}}^{\prime}(\boldsymbol{c}) \boldsymbol{p}_{\boldsymbol{w}}^{\prime}(\boldsymbol{c})\right)$ when $u v w \in H^{(t)}$ : Our goal is to prove (29).

If $c \in A^{(t-1)}(u) \cup A^{(t-1)}(v) \cup A^{(t-1)}(w)$ then $p_{u}^{\prime}(c) p_{v}^{\prime}(c) p_{w}^{\prime}(c)=0=p_{u}(c) p_{v}(c) p_{w}(c)$. Assume then that $c \notin A^{(t-1)}(u) \cup A^{(t-1)}(v) \cup A^{(t-1)}(w)$. If Case B of (3) occurs for $v$ and $w$ then $\left.\mathrm{E}\left(p_{u}^{\prime}(c) p_{v}^{\prime}(c) p_{w}^{\prime}(c)\right)=E\left(p_{u}^{\prime}(c)\right) p_{v}(c)\right) p_{w}(c)$. This is because in Case $\mathrm{B}$, the value of $\eta_{w}(c)$, is independent of all other random variables and so we may use (2). So let us assume that at least two of $p_{u}^{\prime}(c), p_{v}^{\prime}(c), p_{w}^{\prime}(c)$ are both determined according to Case A. Let us in 
fact assume that all three of them are determined by Case A. The case where only two are so determined is similar. Now $\left.p_{u}^{\prime}(c) p_{v}^{\prime}(c) p_{w}^{\prime}(c)\right)=0$ unless $c \notin L(u) \cup L(v) \cup L(w)$. Consequently,

$$
\mathrm{E}\left(p_{u}^{\prime}(c) p_{v}^{\prime}(c) p_{w}^{\prime}(c)\right)=\frac{p_{u}(c)}{q_{u}(c)} \cdot \frac{p_{v}(c)}{q_{v}(c)} \cdot \frac{p_{w}(c)}{q_{w}(c)} \cdot \mathrm{P}(c \notin L(u) \cup L(v) \cup L(w)) .
$$

Now

$$
\begin{aligned}
& \mathrm{P}\left(c \notin L(u) \cup L(v) \cup L(w) \mid \gamma_{u}(c)=\gamma_{v}(c)=\gamma_{w}(c)=0\right)= \\
& q_{u}(c) q_{v}(c) q_{w}(c)\left(1-\theta^{2} p_{v}(c) p_{w}(c)\right)^{-1}\left(1-\theta^{2} p_{u}(c) p_{w}(c)\right)^{-1}\left(1-\theta^{2} p_{u}(c) p_{v}(c)\right)^{-1} \leq \\
& q_{u}(c) q_{v}(c) q_{w}(c)\left(1+4 \theta^{2} \hat{p}^{2}\right) .
\end{aligned}
$$

Let us now argue that

$$
\begin{aligned}
& \mathrm{P}\left(c \notin L(u) \cup L(v) \cup L(w) \mid \gamma_{u}(c)+\gamma_{v}(c)+\gamma_{w}(c)>0\right) \leq \\
& \mathrm{P}\left(c \notin L(u) \cup L(v) \cup L(w) \mid \gamma_{u}(c)=\gamma_{v}(c)=\gamma_{w}(c)=0\right)
\end{aligned}
$$

As before, let $\Omega$ denote the probability space of outcomes of the $\gamma$ 's and $\eta$ 's. For each $i, j, k \in\{0,1\}$, define $\Omega_{i, j, k}$ to be the set of outcomes in $\Omega$ such that $\gamma_{u}(c)=i, \gamma_{v}(c)=$ $j, \gamma_{w}(c)=k$. The sets $\Omega_{i, j, k}$ partition $\Omega$. For each $i, j, k$ with $i+j+k>0$, consider the map $f_{i, j, k}: \Omega_{i, j, k} \rightarrow \Omega_{0,0,0}$ which sets each of $\gamma_{u}(c), \gamma_{v}(c), \gamma_{w}(c)$ to 0 . For $x \in\{u, v, w\}$ define $p_{x}^{i}=\theta p_{x}(c)$ if $i=1$ and $1-\theta p_{x}(c)$ if $i=0$. Let $\Omega_{i, j, k}^{\prime}$ be the set of outcomes in $\Omega_{i, j, k}$ in which $c \notin L(u) \cup L(v) \cup L(w)$. Then

$$
\frac{\mathrm{P}\left(\Omega_{i, j, k}\right)}{\mathrm{P}\left(\Omega_{0,0,0}\right)}=\frac{p_{u}^{i} p_{v}^{j} p_{w}^{k}}{p_{u}^{0} p_{v}^{0} p_{w}^{0}}=\frac{\mathrm{P}\left(\Omega_{i, j, k}^{\prime}\right)}{\mathrm{P}\left(f\left(\Omega_{i, j, k}^{\prime}\right)\right.} .
$$

Observe that if $i+j+k>0$, then $f_{i, j, k}\left(\Omega_{i, j, k}^{\prime}\right) \subset \Omega_{0,0,0}^{\prime}$. Indeed, if $c \notin L(u) \cup L(v) \cup L(w)$, then changing a specific $\gamma$ value from 1 to 0 will still leave $c \notin L(u) \cup L(v) \cup L(w)$. Consequently, for each $i, j, k$,

$$
\frac{\mathrm{P}\left(\Omega_{0,0,0}^{\prime}\right)}{\mathrm{P}\left(\Omega_{0,0,0}\right)} \geq \frac{\mathrm{P}\left(f\left(\Omega_{i, j, k}^{\prime}\right)\right)}{\mathrm{P}\left(\Omega_{i, j, k}\right)} \cdot \frac{\mathrm{P}\left(\Omega_{i, j, k}\right)}{\mathrm{P}\left(\Omega_{0,0,0}\right)}=\frac{\mathrm{P}\left(\Omega_{i, j, k}^{\prime}\right)}{\mathrm{P}\left(\Omega_{i, j, k}\right)} .
$$

It is easy to see that this implies (28). We conclude that

$$
\left.\mathrm{E}\left(p_{u}^{\prime}(c) p_{v}^{\prime}(c) p_{w}^{\prime}(c)\right) \leq p_{u}(c) p_{v}(c) p_{w}(c)\right)\left(1+4 \theta^{2} \hat{p}^{2}\right) .
$$

Estimate for $\mathbf{E}\left(e_{u v}^{\prime}(c) \times \mathbf{1}_{\boldsymbol{u}, \boldsymbol{v} \in U^{\prime}}\right)$ when $u v \in G$ and $\kappa(u v)=c$ : Our goal is to prove

$$
\mathrm{E}\left(e_{u v}^{\prime}(c) \times 1_{u, v \in U^{\prime}}\right) \leq e_{u v}(c)(1+3 \theta \hat{p}) .
$$


If $c \in A^{(t-1)}(u) \cup A^{(t-1)}(v)$ then $e_{u v}^{\prime}(c)=0=e_{u v}(c)$. Assume then that $c \notin A^{(t-1)}(u) \cup$ $A^{(t-1)}(v)$. If Case B of (3) occurs for either $u$ or $v$ then $\mathrm{E}\left(e_{u v}^{\prime}(c)\right)=e_{u v}(c)$. This is because in Case B, the value of $\eta_{u}(c)$ say, is independent of all other random variables and we may use (2). So let us assume that $p_{u}^{\prime}(c), p_{v}^{\prime}(c)$ are both determined according to Case A. Then $e_{u v}^{\prime}(c)=0$ unless $c \notin L(u)$ and $c \notin L(v)$. Consequently,

$$
\begin{aligned}
& \mathrm{E}\left(e_{u v}^{\prime}(c) \times 1_{u, v \in U^{\prime}}\right) \\
& =\frac{p_{u}(c)}{q_{u}(c)} \cdot \frac{p_{v}(c)}{q_{v}(c)} \cdot \mathrm{P}\left(c \notin L(u) \cup L(v) \wedge u, v \in U^{\prime}\right) \\
& \leq \frac{p_{u}(c)}{q_{u}(c)} \cdot \frac{p_{v}(c)}{q_{v}(c)} \cdot \mathrm{P}(c \notin L(u) \cup L(v)) \\
& \leq \frac{p_{u}(c)}{q_{u}(c)} \cdot \frac{p_{v}(c)}{q_{v}(c)} \cdot \mathrm{P}\left(c \notin L(u) \cup L(v) \mid \gamma_{u}(c)=\gamma_{v}(c)=0\right) \\
& \leq p_{u}(c) p_{v}(c)(1-\theta \hat{p})^{-2} \\
& \leq(1+3 \theta \hat{p}) p_{u}(c) p_{v}(c)
\end{aligned}
$$

Explanation: Equation (31) follows as for (28). Equation (32) now follows because the events $c \notin L(u), c \notin L(v)$ become conditionally independent. And then $\mathrm{P}(c \notin L(u) \mid$ $\left.\gamma_{u}(c)=0\right)$ gains a factor $\left(1-\theta p_{v}(c)\right)^{-1} \leq(1-\theta \hat{p})^{-1}$.

\subsection{Proof of (13)}

Given the $p_{u}(c)$ we see that if $Z^{\prime}=\sum_{c \in C} p_{u}^{\prime}(c)$ then $\mathrm{E}\left(Z^{\prime}\right)=\sum_{c \in C} p_{u}(c)$. This follows on using (2). By color independence $Z^{\prime}$ is the sum of $q$ independent non-negative random variables each bounded by $\hat{p}$. Applying (23) we see that

$$
\mathrm{P}\left(\left|Z^{\prime}-\mathrm{E}\left(Z^{\prime}\right)\right| \geq \rho\right) \leq 2 \exp \left\{-\frac{2 \rho^{2}}{q \hat{p}^{2}}\right\}=2 e^{-2 \rho^{2} \Delta^{11 / 12-1 / 2-o(1)}} .
$$

We take $\rho=\Delta^{-1 / 9}$ to see that $\mathcal{E}_{13}(u)$ holds with high enough probability.

\subsection{Proof of (14)}

Let $e_{u v w}(c)=p_{u}(c) p_{v}(c) p_{w}(c)$. Given the $p_{u}(c)$ we see that by $(29), e_{u v w}^{\prime}$ has expectation no more than $e_{u v w}\left(1+4 \theta^{2} \hat{p}^{2}\right)$ and is the sum of $q$ independent non-negative random variables, each of which is bounded by $\hat{p}^{3}$. We have used color independence again here. Applying (23) we see that

$$
\mathrm{P}\left(e_{u v w}^{\prime} \geq e_{u v w}\left(1+4 \theta^{2} \hat{p}^{2}\right)+\rho / 2\right) \leq \exp \left\{-\frac{\rho^{2}}{2 q \hat{p}^{6}}\right\} \leq e^{-\rho^{2} \Delta^{11 / 4-1 / 2-o(1)}}
$$


We also have

$$
4 e_{u v w} \theta^{2} \hat{p}^{2} \leq 4\left(\frac{\omega}{\Delta}+\frac{t}{\Delta^{10 / 9}}\right) \theta^{2} \hat{p}^{2}=\frac{4 \omega \theta^{2} \hat{p}^{2}}{\Delta}+\frac{4 t \theta^{2} \hat{p}^{2}}{\Delta^{10 / 9}}<\frac{1}{2 \Delta^{10 / 9}} .
$$

We take $\rho=\Delta^{-10 / 9}$ to obtain

$$
\mathrm{P}\left(e_{u v w}^{\prime} \geq e_{u v w}+\Delta^{-10 / 9}\right) \leq e^{-\Delta^{\Omega(1)}}
$$

and so $\mathcal{E}_{14}(u)$ holds with high enough probability.

\subsection{Proof of (15)}

Recall that

$$
f_{u}=\sum_{c \in C} \sum_{v \in N(u)} 1_{\kappa(u v)=c} p_{u}(c) p_{v}(c)
$$

If $u v \notin G$ then $\kappa(u v)$ is defined to be $0 \notin C$.

So,

$$
\begin{aligned}
f_{u}^{\prime}-f_{u} & =\sum_{c \in C} \sum_{v \in N(u)}\left(1_{\kappa^{\prime}(u v)=c} p_{u}^{\prime}(c) p_{v}^{\prime}(c)-1_{\kappa(u v)=c} p_{u}(c) p_{v}(c)\right) \\
& =D_{1}+D_{2}
\end{aligned}
$$

where

$$
\begin{aligned}
D_{1} & =\sum_{c \in C} \sum_{\substack{v \in N(u) \\
\kappa(u v)=c}}\left(1_{\kappa^{\prime}(u v)=c} p_{u}^{\prime}(c) p_{v}^{\prime}(c)-p_{u}(c) p_{v}(c)\right) \\
D_{2} & =\sum_{c \in C} \sum_{\substack{v \in N(u) \\
\kappa(u v)=0}} 1_{\kappa^{\prime}(u v)=c} p_{u}^{\prime}(c) p_{v}^{\prime}(c)
\end{aligned}
$$

Here $D_{1}$ accounts for the contribution from edges leaving $G$ and $D_{2}$ accounts for the contribution from edges entering $G$.

We bound $\mathrm{E}\left(D_{1}\right), \mathrm{E}\left(D_{2}\right)$ separately.

$\mathrm{E}\left(D_{1}\right)$ :

$$
\begin{aligned}
D_{1} & =\sum_{c \in C} \sum_{\substack{v \in N(u) \\
\kappa(u v)=c}}\left(1_{\kappa^{\prime}(u v)=c} p_{u}^{\prime}(c) p_{v}^{\prime}(c)-p_{u}(c) p_{v}(c)\right) \\
& =\sum_{c \in C} \sum_{\substack{v \in N(u) \\
\kappa(u v)=c \\
\kappa^{\prime}(u v)=c}}\left(p_{u}^{\prime}(c) p_{v}^{\prime}(c)-p_{u}(c) p_{v}(c)\right)-\sum_{\substack { c \in C \\
\begin{subarray}{c}{v \in N(u) \\
\kappa(u v)=c \\
\kappa^{\prime}(u v) \neq c{ c \in C \\
\begin{subarray} { c } { v \in N ( u ) \\
\kappa ( u v ) = c \\
\kappa ^ { \prime } ( u v ) \neq c } }\end{subarray}} p_{u}(c) p_{v}(c) .
\end{aligned}
$$


Now suppose that $v \notin U^{\prime}$. This means that $v$ has been colored in the current round and so $u v \notin G^{\prime}$. In particular, $\kappa^{\prime}(u v) \neq c$. Therefore the prior expression is bounded from above by

$$
-D_{1,1}+D_{1,2}
$$

where

$$
\begin{aligned}
D_{1,1} & =\sum_{c \in C} \sum_{\substack{v \in N(u) \\
\kappa(u v)=c}} p_{u}(c) p_{v}(c) 1_{v \notin U^{\prime}} \\
D_{1,2} & =\sum_{c \in C} \sum_{\substack{v \in N(u) \\
\kappa(u v)=c}}\left(p_{u}^{\prime}(c) p_{v}^{\prime}(c)-p_{u}(c) p_{v}(c)\right) \times 1_{u, v \in U^{\prime}}
\end{aligned}
$$

Suppose that $x \notin U$ and $u v x \in H$ and $\kappa(x)=c$. Recall that

$$
C_{u}(v)=\left\{c \in C: \gamma_{u}(c)=1\right\} \cup L(v) \cup B(v) .
$$

If there is a tentatively activated color $c^{*}$ at $v$ (i.e. $\gamma_{v}\left(c^{*}\right)=1$ ) that lies outside $C_{u}(v) \cup\{c\}$, then $c^{*} \in \Psi(v)$ and $v$ will be colored in this round. Therefore

$$
\mathrm{P}\left(v \notin U^{\prime} \mid \mathcal{C}\right) \geq \mathrm{P}\left(\exists c^{*} \notin C_{u}(v) \cup\{c\}: \gamma_{v}\left(c^{*}\right)=1 \mid \mathcal{C}\right) .
$$

We have introduced the conditioning $\mathcal{C}$ because we will need it later when we prove concentration.

So by inclusion-exclusion and the independence of the $\gamma_{v}\left(c^{*}\right)$ we can write

$$
\begin{aligned}
\mathrm{E}\left(1_{v \notin U^{\prime}} \mid \mathcal{C}\right) & \geq \mathrm{P}\left(\exists c^{*} \notin C_{u}(v) \cup\{c\}: \gamma_{v}\left(c^{*}\right)=1 \mid \mathcal{C}\right) \\
& \geq \sum_{c^{*} \notin C_{u}(v) \cup\{c\}} \mathrm{P}\left(\gamma_{v}\left(c^{*}\right)=1 \mid \mathcal{C}\right)-\frac{1}{2} \sum_{c_{1}^{*} \neq c_{2}^{*} \notin C_{u}(v) \cup\{c\}} \mathrm{P}\left(\gamma_{v}\left(c_{1}^{*}\right)=\gamma_{v}\left(c_{2}^{*}\right)=1 \mid \mathcal{C}\right) \\
& \geq \sum_{c^{*} \notin C_{u}(v) \cup\{c\}} \theta p_{v}\left(c^{*}\right)-\frac{1}{2}\left(\sum_{c^{*} \notin C_{u}(v) \cup\{c\}} \theta p_{v}\left(c^{*}\right)\right)^{2}
\end{aligned}
$$

Now

$$
\begin{aligned}
\sum_{c^{*} \notin C_{u}(v) \cup\{c\}} \theta p_{v}\left(c^{*}\right) & =\sum_{c^{*} \in C} \theta p_{v}\left(c^{*}\right)-\sum_{c^{*} \in C_{u}(v)} \theta p_{v}\left(c^{*}\right)-\theta p_{v}(c) \\
& \geq \theta\left(\left(1-t \Delta^{-1 / 8}\right)-p_{v}\left(C_{u}(v)\right)-\hat{p}\right) \\
& >\theta\left(1-p_{v}\left(C_{u}(v)\right)-\varepsilon / 2\right)
\end{aligned}
$$


where we have used (6). Also by (6) and the definition of $\hat{p}$ we have

$$
\sum_{c \neq c^{*}} p_{v}\left(c^{*}\right) \leq 1+\Delta^{-1 / 9}<1.1
$$

Consequently

$$
\frac{1}{2}\left(\sum_{c^{*} \notin C_{u}(v) \cup\{c\}} \theta p_{v}\left(c^{*}\right)\right)^{2}=\frac{\theta^{2}}{2}\left(\sum_{c^{*} \notin C_{u}(v) \cup\{c\}} p_{v}\left(c^{*}\right)\right)^{2} \leq \frac{2 \theta^{2}}{3}<\frac{\theta \varepsilon}{2} .
$$

Putting these facts together yields

$$
\mathrm{E}\left(1_{v \notin U^{\prime}} \mid \mathcal{C}\right) \geq \theta\left(1-p_{v}\left(C_{u}(v)\right)-\varepsilon\right) .
$$

Consequently

$$
\mathrm{E}\left(D_{1,1} \mid \mathcal{C}\right) \geq \sum_{c \in C} \sum_{\substack{v \in N(u) \\ \kappa(u v)=c}} p_{u}(c) p_{v}(c) \theta\left(1-p_{v}\left(C_{u}(v)\right)-\varepsilon\right)=\theta\left(1-p_{v}\left(C_{u}(v)\right)-\varepsilon\right) f_{u} .
$$

So,

$$
\mathrm{E}\left(D_{1,1} \mid \mathcal{C}\right) \geq \theta(1-6 \varepsilon) f_{u}, \quad \text { for } \mathcal{C} \text { such that } \mathcal{E}_{(26)} \text { occurs. }
$$

We now consider $D_{1,2}$.

It follows from (8) that $f_{u}<3 \omega$. Together with (30), this gives

$$
\begin{aligned}
\mathrm{E}\left(D_{1,2}\right) & =\sum_{\substack { c \in C \\
\begin{subarray}{c}{v \in N(u) \\
\kappa(u v)=c{ c \in C \\
\begin{subarray} { c } { v \in N ( u ) \\
\kappa ( u v ) = c } }\end{subarray}} \mathrm{E}\left(\left(p_{u}^{\prime}(c) p_{v}^{\prime}(c)-p_{u}(c) p_{v}(c)\right) \times 1_{u, v \in U^{\prime}}\right. \\
& \leq 3 \theta \hat{p} f_{u} \\
& \leq 9 \varepsilon \hat{p} .
\end{aligned}
$$

$\mathrm{E}\left(D_{2}\right)$ :

First observe that

$$
D_{2}=\sum_{c \in C} \sum_{u v_{1} v_{2} \in H^{(t)}}\left(1_{\kappa_{u v_{1}}^{\prime}(c)=1} p_{u}^{\prime}(c) p_{v_{1}}^{\prime}(c)+1_{\kappa_{u v_{2}}^{\prime}(c)=1} p_{u}^{\prime}(c) p_{v_{2}}^{\prime}(c)\right) .
$$

Fix an edge $u v w \in H^{(t)}$. If $w$ is colored with $c$ in this round, then certainly $c$ must have been tentatively activated at $w$. Therefore

$$
\begin{aligned}
\mathrm{E}\left(1_{\kappa^{\prime}(w)=c} p_{u}^{\prime}(c) p_{v}^{\prime}(v)\right) & \leq \mathrm{E}\left(1_{\gamma_{w}(c)=1} p_{u}^{\prime}(c) p_{v}^{\prime}(v)\right) \\
& \leq \theta p_{w}(c) \frac{p_{u}(c)}{q_{u}(c)} \frac{p_{v}(c)}{q_{v}(c)} \mathrm{P}\left(c \notin L(u) \cup L(v) \mid \gamma_{w}(c)=1\right) \\
& \leq \theta p_{w}(c) \frac{p_{u}(c)}{q_{u}(c)} \frac{p_{v}(c)}{q_{v}(c)} \mathrm{P}(c \notin L(u) \cup L(v)) \\
& \leq \theta p_{w}(c) p_{u}(c) p_{v}(c)\left(1+4 \theta^{2} \hat{p}^{2}\right) .
\end{aligned}
$$


We use the argument for (28) to obtain (36) and the argument for (27) to obtain (37).

Going back to (37) we see that

$$
\mathrm{E}\left(D_{2}\right) \leq 2 \theta e_{u}\left(1+4 \theta^{2} \hat{p}^{2}\right)
$$

\subsubsection{Concentration}

We first deal with $D_{1,1}$. For this we condition on the values $\gamma_{w}(c), \eta_{w}(c)$ for all $c \in C$ and all $w \notin N(u)$ and for $w=u$. Then by conditional neighborhood independence $D_{1,1}$ is the sum of at most $\Delta$ independent random variables of value at most $\hat{p}^{2}$. So, for $\rho>0$,

$$
\mathrm{P}\left(D_{1,1}-\mathrm{E}\left(D_{1,1} \mid \mathcal{C}\right) \leq-\rho \mid \mathcal{C}\right) \leq \exp \left\{-\frac{2 \rho^{2}}{\Delta \hat{p}^{4}}\right\}=e^{-\rho^{2} \Delta^{5 / 6-o(1)}}
$$

So, by (34),

$$
\begin{aligned}
& \mathrm{P}\left(D_{1,1} \leq \theta(1-13 \varepsilon / 2) f_{u}-\Delta^{-1 / 8}\right) \\
& =\sum_{\mathcal{C}} \mathrm{P}\left(D_{1,1} \leq \theta(1-13 \varepsilon / 2) f_{u}-\Delta^{-1 / 8} \mid \mathcal{C}\right) \mathrm{P}(\mathcal{C}) \\
& \leq \sum_{\mathcal{C}: \mathcal{E}_{(26) \text { occurs }}} \mathrm{P}\left(D_{1,1} \leq \theta(1-13 \varepsilon / 2) f_{u}-\Delta^{-1 / 8} \mid \mathcal{C}\right) \mathrm{P}(\mathcal{C})+\mathrm{P}\left(\neg \mathcal{E}_{(26)}\right) \\
& \leq \sum_{\mathcal{C}: \mathcal{E}_{(26) \text { occurs }}} \mathrm{P}\left(D_{1,1} \leq \mathrm{E}\left(D_{1,1} \mid \mathcal{C}\right)-\theta \varepsilon f_{u} / 2-\Delta^{-1 / 8} \mid \mathcal{C}\right) \mathrm{P}(\mathcal{C})+\mathrm{P}\left(\neg \mathcal{E}_{(26)}\right) \\
& \leq e^{-\Delta^{5 / 6-o(1)}}+e^{-\Delta^{1 / 12-o(1)}} \\
& =e^{-\Delta^{1 / 12-o(1)}}
\end{aligned}
$$

Now consider the sum $D_{1,2}$. Let $a_{c}=|\{v \in N(u): \kappa(u v)=c\}|$. Note that (11) implies $a_{c} \leq \Delta_{0}=2 t_{0} \Delta \theta \hat{p}$ and note also that $\sum_{c} a_{c} \leq \Delta$. These inequalities give $\sum_{c} a_{c}^{2} \leq \Delta_{0} \Delta$. By color independence, $D_{1,2}$ is the sum of $q$ independent random variables

$$
Y_{c}=\sum_{\substack{v \in N(u) \\ \kappa(u v)=c}}\left(p_{u}^{\prime}(c) p_{v}^{\prime}(c)-p_{u}(c) p_{v}(c)\right)
$$

where $\left|Y_{c}\right| \leq a_{c} \hat{p}^{2}$. So, for $\rho>0$,

$$
\mathrm{P}\left(D_{1,2}-\mathrm{E}\left(D_{1,2}\right) \geq \rho\right) \leq \exp \left\{-\frac{2 \rho^{2}}{\sum_{c} a_{c}^{2} \hat{p}^{4}}\right\} \leq \exp \left\{-\frac{2 \rho^{2}}{\Delta \Delta_{0} \hat{p}^{4}}\right\} \leq e^{-\rho^{2} \Delta^{7 / 24+o(1)}} .
$$

We take $\rho=\Delta^{-1 / 8}$ and use (35) to see that $\mathrm{P}\left(D_{1,2} \geq 2 \Delta^{-1 / 8}\right) \leq e^{-\Delta^{1 / 24-o(1)}}$. Combining this with (38) we see that

$$
\begin{aligned}
\mathrm{P}\left(D_{1} \geq-\theta(1-7 \varepsilon) f_{u}+3 \Delta^{-1 / 8}\right) & \leq \mathrm{P}\left(D_{1,1} \leq \theta\left(1-\frac{13}{2} \varepsilon\right) f_{u}+\Delta^{-1 / 8}\right)+\mathrm{P}\left(D_{1,2} \geq 2 \Delta^{-1 / 8}\right) \\
& \leq e^{-\Delta^{1 / 24-o(1)}} .
\end{aligned}
$$


We now deal with $D_{2}$. There is a minor problem in that $D_{2}$ is the sum of random variables for which we do not have a sufficiently small absolute bound. These variables do however have a small bound which holds with high probability. There are several ways to use this fact. We proceed as follows: Let

$$
D_{2, c}=\sum_{\substack{u v_{1} v_{2} \in H^{(t)} \\ \kappa\left(u v_{i}\right)=0, i=1,2}}\left(1_{\kappa^{\prime}\left(u v_{1}\right)=c} p_{u}^{\prime}(c) p_{v_{1}}^{\prime}(c)+1_{\kappa^{\prime}\left(u v_{2}\right)=c} p_{u}^{\prime}(c) p_{v_{2}}^{\prime}(c)\right)
$$

and

$$
\hat{D}_{2}=\sum_{c \in C} \min \left\{2 \Delta \hat{p}^{3}, D_{2, c}\right\}
$$

Observe that $\hat{D}_{2}$ is the sum of $q$ independent random variables each bounded by $2 \Delta \hat{p}^{3}$. So, for $\rho>0$,

$$
\mathrm{P}\left(\hat{D}_{2}-\mathrm{E}\left(\hat{D}_{2}\right) \geq \rho\right) \leq \exp \left\{-\frac{\rho^{2}}{2 q \Delta^{2} \hat{p}^{6}}\right\} \leq e^{-\rho^{2} \Delta^{1 / 4+o(1)}} .
$$

We take $\rho=\Delta^{-1 / 10}$ to see that

$$
\mathrm{P}\left(\hat{D}_{2} \geq \mathrm{E}\left(\hat{D}_{2}\right)+\Delta^{-1 / 10}\right) \leq e^{-\Delta^{1 / 21}}
$$

We must of course compare $D_{2}$ and $\hat{D}_{2}$. Now $D_{2} \neq \hat{D}_{2}$ only if there exists $c$ such that $D_{2, c}>2 \Delta \hat{p}^{3}$. The latter implies that at least $\Delta \hat{p}$ of the $\gamma_{v_{i}}(c)$ defining $D_{2, c}$ are one. We now use $(24)$ with $\mathrm{E}(X)=2 \Delta \theta \hat{p}$ and $\alpha=1 /(2 \theta)$. this gives

$$
\mathrm{P}\left(D_{2} \neq \hat{D}_{2}\right) \leq q \mathrm{P}(\operatorname{Bin}(2 \Delta, \theta \hat{p}) \geq \Delta \hat{p}) \leq q(2 e \theta)^{\Delta \hat{p}} .
$$

It follows from (41) and $\hat{D}_{2} \leq D_{2} \leq 2 q \Delta \hat{p}^{2}$ that

$$
\left|\mathrm{E}\left(D_{2}\right)-\mathrm{E}\left(\hat{D}_{2}\right)\right| \leq 2 q \Delta \hat{p}^{2} \mathrm{P}\left(D_{2} \neq \hat{D}_{2}\right) \leq 2 \Delta \hat{p}^{2} q^{2}(2 e \theta)^{\Delta \hat{p}}<(\log \Delta)^{-\Delta^{13 / 24}+o(1)} .
$$

Applying (40) and (41) we see that

$$
\begin{aligned}
& \mathrm{P}\left(D_{2} \geq \mathrm{E}\left(D_{2}\right)+\Delta^{-1 / 20}+2 \Delta \hat{p}^{2} q^{2}(e \theta)^{\Delta \hat{p}}\right) \leq \\
& \mathrm{P}\left(\hat{D}_{2} \geq \mathrm{E}\left(\hat{D}_{2}\right)+\Delta^{-1 / 20}\right)+\mathrm{P}\left(D_{2} \neq \hat{D}_{2}\right) \leq e^{-\Delta^{1 / 21}}+q(2 e \theta)^{\Delta \hat{p}} .
\end{aligned}
$$

Combining this with (39) we see that with probability at least $1-e^{-\Delta^{\Omega(1)}}$,

$$
\begin{aligned}
f_{u}^{\prime}-f_{u} & \\
& \leq-\theta(1-7 \varepsilon) f_{u}+3 \Delta^{-1 / 8}+8 \omega \theta^{2} \hat{p}^{2}+2 \theta e_{u}\left(1+4 \theta^{2} \hat{p}^{2}\right)+\Delta^{-1 / 20}+2 \Delta \hat{p}^{2} q^{2}(e \theta)^{\Delta \hat{p}} \\
& \leq \theta\left(2 e_{u}-(1-7 \varepsilon) f_{u}\right)+\Delta^{-1 / 21} .
\end{aligned}
$$

This confirms (15). 


\subsection{Proof of (16)}

Fix $c$ and write $p^{\prime}=p_{u}^{\prime}(c)=p \delta$. We consider two cases, but in both cases $\mathrm{E}(\delta)=1$ and $\delta$ takes two values, 0 and $1 / \mathrm{P}(\delta>0)$. Then we have

$$
\mathrm{E}\left(-p^{\prime} \log p^{\prime}\right)=-p \log p-p \log (1 / \mathrm{P}(\delta>0)) .
$$

(i) $p=p_{u}(c)$ and $\delta=\gamma_{u}(c) / q_{u}(c)$ and $\gamma_{u}(c)$ is a $\{0,1\}$ random variable with $\mathrm{P}(\delta>0)=$ $q_{u}(c)$.

(ii) $p=p_{u}(c)=\hat{p}$ and $\delta$ is a $\{0,1\}$ random variable with $\mathrm{P}(\delta>0)=p_{u}(c) / \hat{p} \geq q_{u}(c)$.

Thus in both cases

$$
\mathrm{E}\left(-p^{\prime} \log p^{\prime}\right) \geq-p \log p-p \log 1 / q_{u}(c)
$$

Observe next that $0 \leq a, b \leq 1$ implies that $(1-a b)^{-1} \leq(1-a)^{-b}$ and $-\log (1-x) \leq x+x^{2}$ for $0 \leq x \ll 1$. So,

$$
\begin{aligned}
\log 1 / q_{u}(c) & \leq-\sum_{u v w \in H^{(t)}} p_{v}(c) p_{w}(c) \log \left(1-\theta^{2}\right)-\sum_{\substack{u v \in G^{(t)} \\
\kappa(u v)=c}} p_{v}(c) \log (1-\theta) \\
& \leq\left(\theta^{2}+\theta^{4}\right) e_{u}(c)+\left(\theta+\theta^{2}\right) f_{u}(c) .
\end{aligned}
$$

Now

$$
\begin{aligned}
\mathrm{E}\left(h_{u}-h_{u}^{\prime}\right) & \leq \mathrm{E}\left(\sum_{c}-p_{u}(c) \log p_{u}(c)\right)-\mathrm{E}\left(\sum_{c}-p_{u}^{\prime}(c) \log p_{u}^{\prime}(c)\right) \\
& \leq \sum_{c}-p_{u}(c) \log p_{u}(c)-\left(\sum_{c}-p_{u}(c) \log p_{u}(c)-p_{u}(c) \log 1 / q_{u}(c)\right) \\
& =\sum_{c} p_{u}(c) \log 1 / q_{u}(c) \\
& \leq\left(\theta^{2}+\theta^{4}\right) \sum_{c} p_{u}(c) e_{u}(c)+\left(\theta+\theta^{2}\right) \sum_{c} p_{u}(c) f_{u}(c) \\
& =\left(\theta^{2}+\theta^{4}\right) e_{u}+\left(\theta+\theta^{2}\right) f_{u} \\
& \leq\left(\theta^{2}+\theta^{4}\right)\left(\omega+t \Delta^{-1 / 9}\right)(1-\theta / 3)^{t}+3\left(\theta+\theta^{2}\right)(1-\theta / 4)^{t} \omega \\
& \leq 4 \varepsilon(1-\theta / 4)^{t} .
\end{aligned}
$$

Given the $p_{u}(c)$ we see that $h_{u}^{\prime}$ is the sum of $q$ independent non-negative random variables with values bounded by $-\hat{p} \log \hat{p} \leq \Delta^{-11 / 24+o(1)}$. Here we have used color independence. So,

$$
\mathrm{P}\left(h_{u}-h_{u}^{\prime} \geq 4 \varepsilon(1-\theta / 4)^{t}+\rho\right) \leq \exp \left\{-\frac{2 \rho^{2}}{q(\hat{p} \log \hat{p})^{2}}\right\}=e^{-2 \rho^{2} \Delta^{5 / 12-o(1)}} .
$$


We take $\rho=\varepsilon(1-\theta / 4)^{t} \geq(\log \Delta)^{-O(1)}$ to see that $h_{u}-h_{u}^{\prime} \leq 5 \varepsilon(1-\theta / 4)^{t}$ holds with high enough probability.

\subsection{Proof of (17)}

Fix $u$ and condition on the values $\gamma_{w}(c), \eta_{w}(c)$ for all $c \in C$ and all $w \notin N(u)$ and for $w=u$. Now write $u \sim v$ to mean that there exists $w$ such that $u v w$ is an edge of $H^{(t)}$ or that $u v$ is an edge of $G$. Then write

$$
Z_{u}=d(u)-d^{\prime}(u) \geq \frac{1}{2} \sum_{u \sim v} Z_{u, v} \text { where } Z_{u, v}=1_{v \notin U^{\prime}}
$$

Now, for $e=u v w \in H^{(t)}$ let $Z_{u, e}=Z_{u, v}+Z_{u, w}$ and if $e=u v \in G$ let $Z_{u, e}=Z_{u, v}$. Conditional neighborhood independence implies that the collection $Z_{u, e}$ constitute an independent set of random variables. Applying (23) to $Z_{u}=\sum_{e} Z_{u, e}$ we see that

$$
\mathrm{P}\left(Z_{u} \leq \mathrm{E}\left(Z_{u}\right)-\Delta^{2 / 3}\right) \leq \exp \left\{-\frac{2 \Delta^{4 / 3}}{4 \cdot \Delta / 2}\right\}=e^{-\Delta^{1 / 3}}
$$

and so we only have to estimate $\mathrm{E}\left(Z_{u}\right)$.

Fix $v \sim u$. Let $C_{u}(v)$ be as in (26). Condition on $\mathcal{C}$. $v$ is a member of $U^{\prime}$ if none of the colors $\left.c \notin C_{u}(v)\right)$ are tentatively activated. (It is tempting to write iff but this would not be true. If $u v w \in H$ then we could add the effect of those colors which are activated at $u$ and not $w$ to the RHS of $(43) . C_{u}(v)$ contains any of these). The activations we consider are done independently and so

$$
\begin{aligned}
\mathrm{P}\left(v \in U^{\prime} \mid \mathcal{C}\right) & \leq \prod_{c \notin C_{u}(v)}\left(1-\theta p_{v}(c)\right) \\
& \leq \exp \left\{-\sum_{c \notin C_{u}(v)} \theta p_{v}(c)\right\} \\
& \leq \exp \left\{-\theta\left(1-\Delta^{-1 / 9}\right)+\theta p_{v}\left(C_{u}(v)\right)\right\}
\end{aligned}
$$

If $\mathcal{E}_{(26)}$ occurs then $p_{v}\left(C_{u}(v)\right) \leq 5 \varepsilon$. Consequently,

$$
\begin{aligned}
\mathrm{P}\left(v \notin U^{\prime}\right) & \geq \sum_{\mathcal{C}: \mathcal{E}_{(26)} \text { occurs }}\left(1-\exp \left\{-\theta\left(1-\Delta^{-1 / 9}\right)+5 \theta \varepsilon\right\}\right) \mathrm{P}(\mathcal{C}) \\
& \geq 6 \theta / 7 .
\end{aligned}
$$

This gives

$$
\mathrm{E}\left(Z_{u}\right) \geq \frac{3}{7} \theta d(u)
$$




\subsection{Proof of (18)}

Observe that if $u w \in G^{\prime} \backslash G$ and $\kappa^{\prime}(u w)=c$ then there must exist a vertex $v$ and an edge $u v w \in H^{(t)}$ such that $v$ gets colored in Step $t$. In particular we must have $\gamma_{v}(c)=1$. Hence,

$$
d_{G^{\prime}}(u, c)-d_{G}(u, c) \leq \sum_{u \sim v} 1_{\gamma_{v}(c)=1}
$$

is bounded by the sum of $\Delta$ independent $0-1$ random variables each having expectation at most $\theta \hat{p}$. Therefore

$$
\mathrm{P}\left(d_{G^{\prime}}(u, c)-d_{G}(u, c) \geq 2 \Delta \theta \hat{p}\right) \leq e^{-\Delta \theta \hat{p} / 3} .
$$

\section{List Coloring}

Here we describe the small modifications needed to our argument to prove the same result for list colorings. Each vertex $v \in V$ starts with a set $A_{v}$ of $2 q$ available colors. Choose for each $v$ a set $B_{v} \subseteq A_{v}$ where $\left|B_{v}\right|=q$. Let now $C=\bigcup_{v \in V} B_{v}$. We initialise $p_{v}(c)=$ $q^{-1} 1_{c \in B_{v}}$ and follow the main argument as before. When the semi-random procedure finishes, the local lemma can be used to show that the lists $A_{v} \backslash B_{v}$ can be used to color the vertices that remain uncolored.

\section{Acknowledgments}

We thank a referee for helpful comments.

\section{References}

[1] M. Ajtai, J. Komlós, J. Pintz, J. Spencer, E. Szemerédi, Extremal uncrowded hypergraphs, J. Combin. Theory Ser. A 32 (1982), no. 3, 321-335.

[2] N. Alon, M. Krivelevich, B. Sudakov, Coloring graphs with sparse neighborhoods, J. Combin. Theory Ser. B 77 (1999), no. 1, 73-82.

[3] T. Bohman, A. Frieze, D. Mubayi, Coloring $H$-free hypergraphs, submitted. Pre-print available at http://www.math.cmu.edu/ af1p/Texfiles/Hypchrom.pdf.

[4] W. T. Gowers, Hypergraph regularity and the multidimensional Szemerédi theorem, Ann. of Math. (2) 166 (2007), no. 3, 897-946. 
[5] A. Johansson, Asymptotic choice number for triangle free graphs, DIMACS Technical Report 91-4, 1196.

[6] J. Kahn, Asymptotically good list-colorings, J. Combin. Theory Ser. A 73 (1996), no. $1,1-59$.

[7] J. H. Kim, On Brooks' theorem for sparse graphs, Combin. Probab. Comput. 4 (1995), no. 2, 97-132.

[8] J. Komlós, J Pintz, E. Szemerédi, A lower bound for Heilbronn's problem, J. London Math. Soc. (2) 25 (1982), no. 1, 13-24.

[9] A. Kostochka, V. Rödl, On Ramsey numbers of uniform hypergraphs with given maximum degree, J. Combin. Theory Ser. A 113 (2006), no. 7, 1555-1564.

[10] M. Krivelevich, Bounding Ramsey numbers through large deviation inequalities, Random Structures Algorithms 7 (1995), no. 2, 145-155.

[11] B. Nagle, V. Rödl, M. Schacht, The counting lemma for regular $k$-uniform hypergraphs, Random Structures and Algorithms 28 (2006), no. 2, 113-179.

[12] V. Rödl, On a packing and covering problem, European J. Combin. 6 (1985), no. 1, 69-78.

[13] V. Rödl, A. Ruciński, E. Szemerédi, Endre, A Dirac-type theorem for 3-uniform hypergraphs, Combin. Probab. Comput. 15 (2006), no. 1-2, 229-251.

[14] T. Tao, A variant of the hypergraph removal lemma, J. Combin. Theory Ser. A 113 (2006), no. 7, 1257-1280. 